\title{
Micro- and Nanoprocessing of Polymers Using a Laser Plasma Extreme Ultraviolet Source
}

\author{
A. Bartnik, H. Fiedorowicz, R. Jarocki, J. Kostecki, R. Rakowski, A. Szczurek \\ AND M. SZCZUREK
}

Institute of Optoelectronics, Military University of Technology, Kaliskiego 2, 00-908 Warsaw, Poland

Laser plasma with temperature of the order of tens $\mathrm{eV}$ can be an efficient source of extreme ultraviolet (EUV). The radiation can be focused using different kind of optics, giving sufficient fluence for some applications. In this work we present results of investigations concerning applications of a laser plasma EUV source based on a double stream gas puff target. The source was equipped with two different grazing incidence collectors. One of them was a multifoil collector, the second one was an axisymmetrical ellipsoidal collector. The multifoil mirror was used mainly in experiments concerning micromachining of organic polymers by direct photo-etching. The experiments were performed for different polymers that were irradiated through a fine metal grid as a contact mask. The smallest element of a pattern structure obtained in this way was $5 \mu \mathrm{m}$, while the structure height was $50 \mu \mathrm{m}$ giving an aspect ratio about 10. The laser-plasma EUV source equipped with the axisymmetrical ellipsoidal collector was used for surface modification of organic polymers and inorganic solids. The surface morphology after irradiation was investigated. Different forms of micro- and nanostructures were obtained depending on material and irradiation conditions.

PACS numbers: $52.38 . \mathrm{Ph}, 82.50 . \mathrm{Kx}, 81.40 . \mathrm{Wx}$

\section{Introduction}

It was shown in many publications that soft X-ray (SXR) or EUV radiation can be utilized for direct photo-etching and surface modification of different materials. In the case of polymers a single photon is capable to break many molecular bonds of a polymer chain. Some fragments of the chain can then evaporate from the polymer surface. Using synchrotron radiation Zhang et al. showed that such photo-etching process can be applied for direct micromachining of polymers [1]. They showed that the method allows to obtain microstructures with high aspect ratio. An ablation of the material, in this case, is not connected with thermal effects allowing to obtain sharp edges. A series of similar experiments with laser-plasma SXR and EUV sources instead of synchrotron was performed by authors of this paper. The experiments were carried out using a single shot, high power laser at Prague Asterix Laser System (PALS) [2-4] and also, with the use of a $10 \mathrm{~Hz}$ laser-plasma radiation source [5]. Photo-etching of inorganic materials is also possible but requires higher EUV fluence in relation to polymers. Some experiments with photo-etching of inorganic materials, using laser-plasma radiation, were performed by Makimura et al. [6]. They used Ta laser plasma to create pattern in a silica glass. In this case, the fluence was estimated to be $0.1 \mathrm{~J} / \mathrm{cm}^{2}$. Photo-etching of materials requires exceeding of an ablation threshold fluence. The higher fluence is the higher ablation rate per pulse and lower surface roughness after irradiation are. In some applications however, a surface roughness in micro- or nanoscale is desirable. Modification of surface topography is especially interesting in the case of organic polymers. Such modification accompanied by some chemical changes is very important in a wide spectrum of biotechnologies. A very important issue here is a biocompatibility of polymers. It is connected with the cell adhesion and proliferation on the polymer surface. It strongly depends on chemical and physical properties of the surface. Various surface structures in micro and nanoscale were applied to study correlation between surface topography and cell adhesion [7-9]. Chemical modifications of polymers are based on the incorporation of new chemical groups at their surfaces. It can be achieved using various chemical and biological methods, however, the most promising are physical methods, such as ion implantation or UV irradiation in reactive atmosphere $[10,11]$. It seems that either physical or chemical surface modification can be also obtained using EUV radiation. It could have some advantages connected with a very short absorption length of EUV photons in any material. Such investigations were undertaken by authors of this paper using a laser-plasma EUV source.

In this work we present results of experiments connected with micromachining of polymers and surface modification of organic and inorganic materials using EUV pulses. Experimental results were obtained using a $10 \mathrm{~Hz}$ EUV source based on a laser-irradiated gas puff target [12]. Scanning electron microscopy (SEM) and atomic force microscopy (AFM) images of surface structures in micro- and nanoscale are presented. The most interesting structures were obtained for selected polymers. 


\section{Laser-plasma EUV source}

In the experiments, a $10 \mathrm{~Hz}$ laser plasma EUV source based on a double stream gas puff target was used. The target was irradiated with 4 ns Nd:YAG laser pulses with energy of $0.8 \mathrm{~J}$. Laser pulses were focused using a single spherical bi-convex lens with a focal length of $25 \mathrm{~mm}$. The target was placed $1 \mathrm{~mm}$ out of focus. The focal spot size at this position was approximately $300 \mu \mathrm{m}$. The double stream gas puff target was developed at the Institute of Optoelectronics [13]. The target was created by pulsed injection of xenon or krypton into a hollow stream of helium with the use of an electromagnetic valve system equipped with a double nozzle setup. The outer stream of gas (the buffer gas) confines the inner gas stream and prevents its spherical expansion outside the nozzle. This way, the inner gas has a form of an elongated stream with relatively high density and a sharp density gradient at the boundary with the outer gas. It makes possible to focus a laser beam in the high density region of gas, far away from the nozzle outlet and achieve an efficient EUV production without degradation of the nozzle. Apart from that helium surrounding the inner gas has a high ionization potential so, its influence on the laser beam focused at the inner gas is negligible. Thus, a laser plasma is created mainly in the inner gas. Additionally, the ion stream created in the interaction region is suppressed by the cold helium surrounding the plasma. Also, the absorption coefficient of helium in EUV wavelength range is much lower than for gases with high atomic number so, it absorbs only a small fraction of radiation emitted from the plasma. The gas puff target formed in such a way has then many advantages and allows to generate EUV radiation with relatively high efficiency and without target debris. The main disadvantage is the attenuation of EUV in a residual gas filling the vacuum chamber during operation with high repetition. To minimize the absorption, the EUV collector was mounted in an additional chamber and a differential pumping arrangement was applied.

\section{EUV collectors}

\subsection{Multifoil collector}

The grazing incidence EUV optic was prepared for efficient collection of laser-plasma radiation for wavelengths longer than $8 \mathrm{~nm}$. The optic consists of two orthogonal stacks of ellipsoidal mirrors forming a double-focusing device. The ellipsoidal surfaces are covered by a layer of gold that has relatively high reflectivity at the wavelength range between 8-20 $\mathrm{nm}$ up to about 10 degrees of an incidence angle. A schematic view of half of the optics system is shown in Fig. 1. Mirrors forming the device have the width of $40 \mathrm{~mm}$. As can be noticed, the spacing between adjacent mirrors rises with the distance from the symmetry axis. The curvature of the mirrors and the spacing between them were optimized by ray tracing simulations to maximize an aperture of the optic and to minimize the size of the focal spot. Parameters of the focused
EUV radiation were measured using a pinhole camera, an EUV spectrograph and the X-ray detector. Imaging of the focal spot was performed using the pinhole camera coupled to the CCD camera. The EUV radiation scattered in the foil located in the focal plane was registered using a pinhole camera located at an optical axis of the collector. There was no direct radiation along the collector axis because of its mechanical construction so, only the scattered radiation could be registered. Resolution of this method was limited mainly by the pinhole diameter which was $20 \mu \mathrm{m}$. Full width at half maximum (FWHM) of the focal spot, estimated from the image obtained this way, was approximately $1.2 \mathrm{~mm}$.

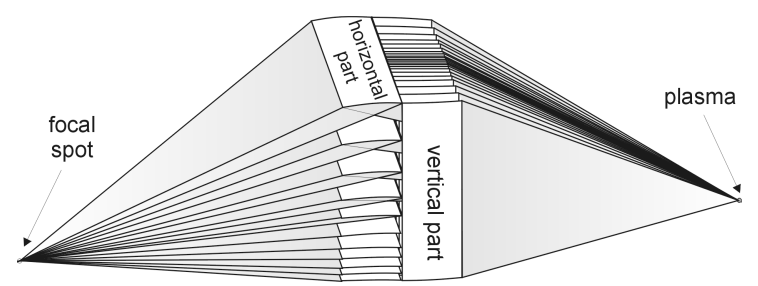

Fig. 1. Schematic view of half of the multifoil EUV collector.

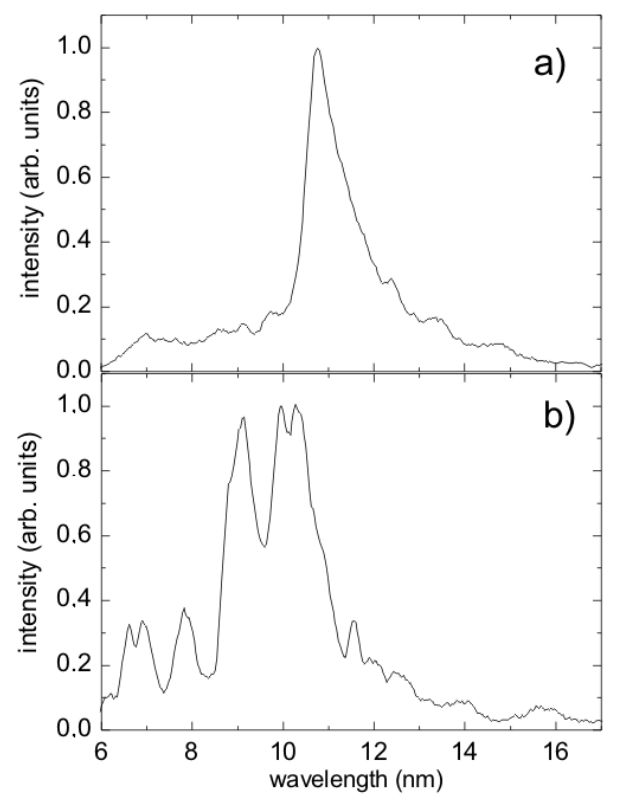

Fig. 2. Spectra of the EUV radiation focused with the multifoil collector: a) xenon plasma, b) krypton plasma.

A similar method was applied to measure spectra of the focused radiation from xenon and krypton plasmas. In this case, a spectrograph with 5000 lines/mm transmission grating was applied instead of the pinhole camera. The spectra shown in Fig. 2 contained a large number of unresolved lines with the dominating feature in the wavelength range about $11 \mathrm{~nm}$ for Xe and $10 \mathrm{~nm}$ for Kr. Influence of $\mathrm{Zr}$ foil on the spectrum was not significant 
in the wavelength range of $7-16 \mathrm{~nm}$, because its transmission in this range is almost constant. A strong attenuation appeared below $6 \mathrm{~nm}$, but in that range even the direct radiation was rather week. So, the main part of radiation spectrum was practically the same as for a direct radiation.

The EUV fluence was measured using a calibrated pinhole located in the focal plane coupled to an AXUV100 detector with a deposited $200 \mathrm{~nm} \mathrm{Zr}$ filter. The pinhole could be moved in the focal plane allowing to find a maximum fluence and to determine its distribution. The fluence distribution measured in this way corresponded to the distribution of the focal image intensity. The maximal estimated value was $30 \mathrm{~mJ} / \mathrm{cm}^{2}$ and $17 \mathrm{~mJ} / \mathrm{cm}^{2}$ for $\mathrm{Xe}$ and $\mathrm{Kr}$, respectively.

\subsection{Ellipsoidal collector}

The second grazing incidence collector was a part of a single ellipsoid coated with a gold having a collection angle $0.75 \mathrm{sr}$. The roughness of its inner surface was better than $1 \mathrm{~nm}$. The incidence angle was about 15 degrees. A schematic view of the optic was shown in Fig. 3. It has a rotational symmetry and plasma is located in one of its focal points. The ellipsoidal mirror is not an imaging optic. It is the reason that cross section of the focused radiation near the second focal point does not depend much on the plasma shape and moreover, it is axisymmetrical. For the same reasons, in the case of such collecting mirror, an exact magnification can not be defined. There can be defined only an average magnification for a circular radiation source. For the collector used in this experiment the average magnification was approximately 2. The plasma size was about $0.5 \mathrm{~mm}$ and the FWHM of focused radiation in the focal plane was approximately $1 \mathrm{~mm}$. The relatively large angle of incidence, in respect to the multifoil optic, resulted in significant differences in the EUV spectra of the focused radiation. In case of the multifoil collector for EUV, spectrally integrated intensity in the wavelength range close to its maximum value dominates. In the case of the ellipsoidal collector, spectrum of the focused Kr radiation consists of a narrow feature the with maximum close to $10 \mathrm{~nm}$ and a long-wavelength tail up to $70 \mathrm{~nm}$. In this case, spectrally integrated intensities in both ranges are comparable. Low and high resolution $\mathrm{Kr}$ spectra of the focused radiation are presented in Fig. 4. The maximal fluence in the wavelength $9-17 \mathrm{~nm}$ range was approximately of $30 \mathrm{~mJ} / \mathrm{cm}^{2}$, while in the wavelength range of 9-70 $\mathrm{nm}$ it exceeded $60 \mathrm{~mJ} / \mathrm{cm}^{2}$.

\section{Experimental results}

\subsection{Micromachining}

First experiments, connected with micromachining of polymers by direct photo-etching, were performed without any EUV optics. The radiation fluence in this case did not overcome $3 \mathrm{~mJ} / \mathrm{cm}^{2}$ and photo-etching of polymers was very weak. In case of polytetrafluoroethylene

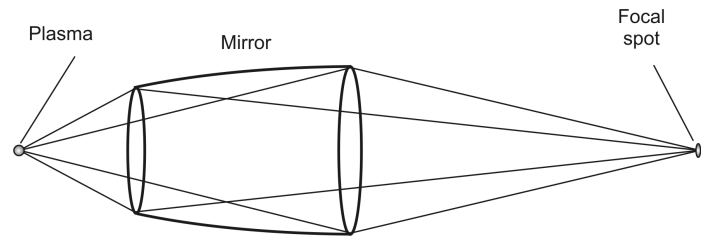

Fig. 3. Schematic view of the ellipsoidal EUV collector.

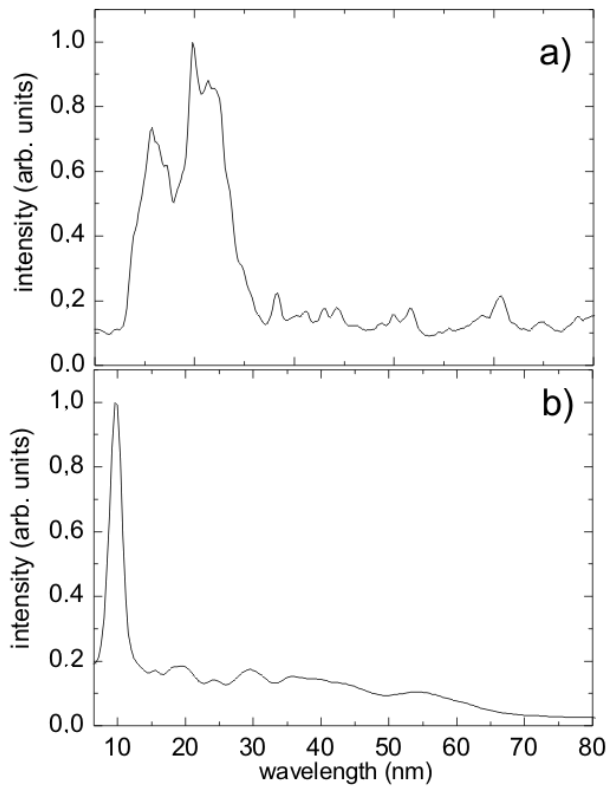

Fig. 4. Spectra of the EUV radiation from Kr plasma focused with the ellipsoidal collector: a) high resolution - close to maximum, b) low resolution - the whole EUV range.

(PTFE) it was of the order of $1 \mu \mathrm{m}$ per $10 \mathrm{~min}$ exposure with $10 \mathrm{~Hz}$ repetition rate at room temperature [5]. It was possible to increase the photo-etching efficiency several times by heating the sample to the temperature over $200{ }^{\circ} \mathrm{C}$ [14]. Application of the multifoil collector allowed to increase an EUV fluence up to $30 \mathrm{~mJ} / \mathrm{cm}^{2}$ in the focal spot. Polymer samples were then mounted in the focal plane and were irradiated through a fine metal grid serving as a contact mask. Experiments were performed for different polymer samples. The samples were irradiated during 2-4 min with a $10 \mathrm{~Hz}$ repetition rate at room temperature. In this case, the EUV fluence was sufficient for photo-etching of any polymer. The best results, however, were obtained for PTFE and fluorinated ethylene-propylene (FEP). Similar photo-etching rate was obtained also for an organic crystal cesium acid phthalate (CsAP). The examples of patterns obtained in $50 \mu \mathrm{m}$ thick PTFE and FEP foils after 4 min irradiation in a central part of the focal spot are presented in Fig. 5a-b. The material was completely removed from areas not covered by metal. The edges are sharp at least in micrometer scale. The photo-etching depth in the CsAP 
crystal was a little bit less reaching about $30 \mu \mathrm{m}$, but the edges are also sharp (Fig. 5c).

a)



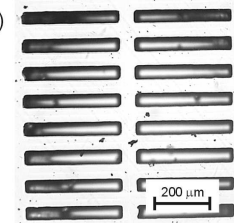

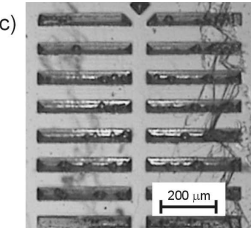

Fig. 5. Examples of microstructures obtained as a result of photoetching of different materials with EUV focused with the multifoil collector: a) PTFE, b) FEP, c) CsAP.
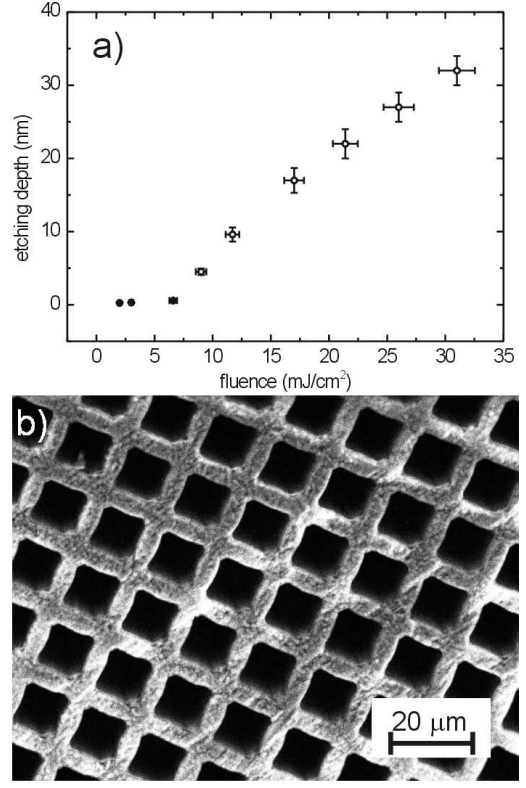

Fig. 6. Photo-etching of PTFE: a) etching depth dependence on the EUV fluence, b) SEM image of a deep microstructure.

A dependence of photo-etching depth per shot versus EUV fluence was determined for PTFE, and it is presented in Fig. 6a. It can be noticed that the ablation depth for fluences within the range of $2-7 \mathrm{~mJ} / \mathrm{cm}^{2}$ is very low and does not change significantly. For greater values, it increases almost linearly with the fluence. The EUV fluence for micromachining should then overcome $7 \mathrm{~mJ} / \mathrm{cm}^{2}$. Another problem is a possibility to create microstructures with a high aspect radio. The possibility with our EUV source was verified using a copper grid as the mask with $5 \mu \mathrm{m}$ bars and $50 \mu \mathrm{m}$ thick PTFE foil as the irradiated sample. As previously, the irradiation was carried out in the multifoil collector focal spot. In this case, the material was completely removed from areas not covered by metal, in the central part of the focal spot. The aspect ratio in this case, defined as a ratio of a microstructure depth to a smallest lateral size, was 10 . SEM picture of the microstructure is shown in Fig. $6 \mathrm{~b}$.

\subsection{Surface modification of polymers}

For surface modifications of polymers, the ellipsoidal collector was employed. The interaction experiments were performed with the use of $50 \mu \mathrm{m}$ thick polymer sheets from Goodfellow. The samples were used without any treatment. It was shown in micromachining experiments that EUV fluence $>7 \mathrm{~mJ} / \mathrm{cm}^{2}$ is sufficient for efficient photo-etching of polytetrafluoroethylene (PTFE), fluorinated ethylene-propylene (FEP) and poly(methyl methacrylate) (PMMA). The maximum value of the EUV fluence in the focal spot of the ellipsoidal mirror was several times higher. It means that photo-etching and some surface changes of these polymers should occur. In case of other polymers at least some surface modifications were expected. A polymer sample for irradiation was mounted in a special holder on the $X Y Z$ translation stage. It was then possible to move it to different positions in respect to the focal point. At first, every polymer sample was irradiated in the focal plane to verify the possibility of its photo-etching. The samples were irradiated typically for $30 \mathrm{~s}-1 \mathrm{~min}$., with $10 \mathrm{~Hz}$ repetition rate. In every case, a hole was created near the centre of the focal spot. It is the evidence of the efficient material ablation in this area. It was estimated that an average thickness of the polymer material removed in one pulse was about $100 \mathrm{~nm}$. The roughness of the surface after ablation depended on the kind of polymer. The PTFE surface after ablation was relatively rough with nonuniformities in the micrometer scale. PMMA and FEP surfaces remained smooth in this scale. However, in every case some conical structures appeared. The structures are presented in Fig. 7. The height of cones is comparable with the thickness of the material removed. Their origin is probably connected with a shielding effect of some surface or volume impurities having a higher ablation threshold than the polymer, as it was suggested in case of UV laser ablation $[15,16]$. The material ablation is limited to some area within the focal spot. Its size is connected with EUV distribution at the surface of irradiated polymer and its ablation threshold. Smooth ablation is possible if the EUV fluence exceeds significantly the ablation threshold value. In the area irradiated with the fluence close to the threshold value, the surface topography changes dramatically. It can be recognized as a ring of a modified surface surrounding the zone of smooth ablation. Typical structures occurring in the rings are presented in Fig. 8. It can be easily noticed that the structures in fluoropolymers have the characteristic scale of the order of hundreds of nanometers, while the structures in PMMA have the scale of the order of a few micrometers. There is no dependence between initial surface roughness and form of the structures after irradiation with the fluence close to the ablation threshold. Surface smoothness of PMMA and FEP before irradiation was similar, but their final structures were completely different. On the other hand, the roughness of PTFE surface at the beginning was much higher than FEP, and the characteristic size of their final structures was similar. However, these 
polymers are fluoropolymers having the same stoichiometric composition in contrary to PMMA, which does not contain fluorine at all. It is probably the main factor influencing the surface structure of polymers. Quite different situation was for other polymers used in the experiment: polyethylene naphthalate (PEN), polyethylene terephthalate (PET), polyvinyl fluoride (PVF), and polyvinylidene chloride (PVDC). In case of these polymers there was no smooth ablation. In contrary, there was a strong surface modification in some area close to the centre of the focal spot. Typical structures are shown in Fig. 9. Surface structures, except this of PVDC, have a pronounced orientation. The formation mechanism of these structures is connected with material ablation and relaxation of frozen stress fields in the polymer, originating from the production process. Surface forms after irradiation depend both on the fluence and the number of shots. The structures shown in Fig. 9 were obtained as a result of irradiation with 25 EUV pulses (only PVDC was irradiated with 10 pulses). Two times longer exposure resulted in structures presented in Fig. 10. It is clearly visible that orientation of the structures is sustained, while the characteristic sizes of the microstructures increase with the number of shots. Increase of the structures is probably a combined effect of nonuniform photoetching and the Marangoni convection of the molten surface layer of polymer. The Marangoni effect is driven by local variation of the surface tension of the fluid, which is due to temperature gradients. The stronger roughness of the surface is, the stronger temperature gradients connected with the EUV irradiation are. On the other hand, interaction of EUV photons with polymers depends on the incidence angle, what means that different forms of the surface structures influence the local absorption the EUV photon. These two effects lead to formation and growth of different kinds of surface microstructures.

\subsection{Interaction with solids}

Inorganic materials usually consist of atoms or relatively simple molecules. Boiling points of chemical elements in a solid state are high with some exceptions. That is why their ablation requires much higher fluence comparing to polymers. In a case of chemical compounds, they can be decomposed giving volatile components. In this case, the ablation is possible even with relatively low EUV fluence. Apart from that, in most cases, melting of a thin layer is possible and thus, some surface modifications can occur. For some chemical compounds either melting or decomposition can appear. It should result in different complicated structures as it took place in case of polymers. Two interesting examples of the resulting structures in the compounds are shown in Fig. 11. First of them (Fig. 11a) is a surface of $\mathrm{NaCl}$ monocrystal after 1 min irradiation with $10 \mathrm{~Hz}$ repetition rate, the second one is a $\mathrm{CaF}_{2}$ monocrystal. The irradiation was performed using the grazing incidence collector. A thin surface layer of the monocrystal was divided into microcrystals. The smallest crystal size shown here was about
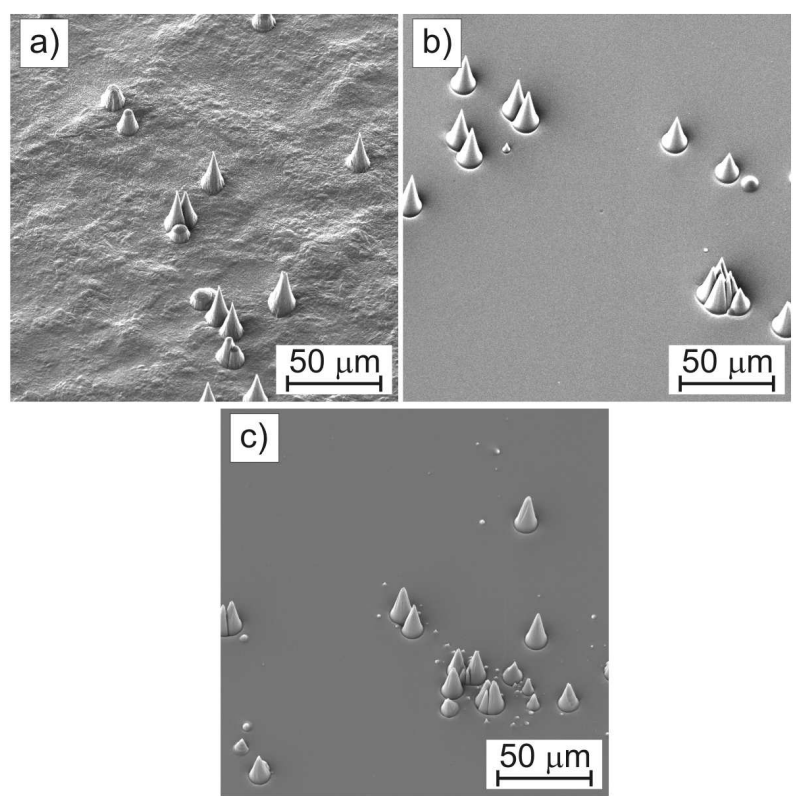

Fig. 7. SEM images of conical structures in polymers after 2 min EUV irradiation with the fluence well above the ablation threshold: a) PTFE, b) FEP, c) PMMA.

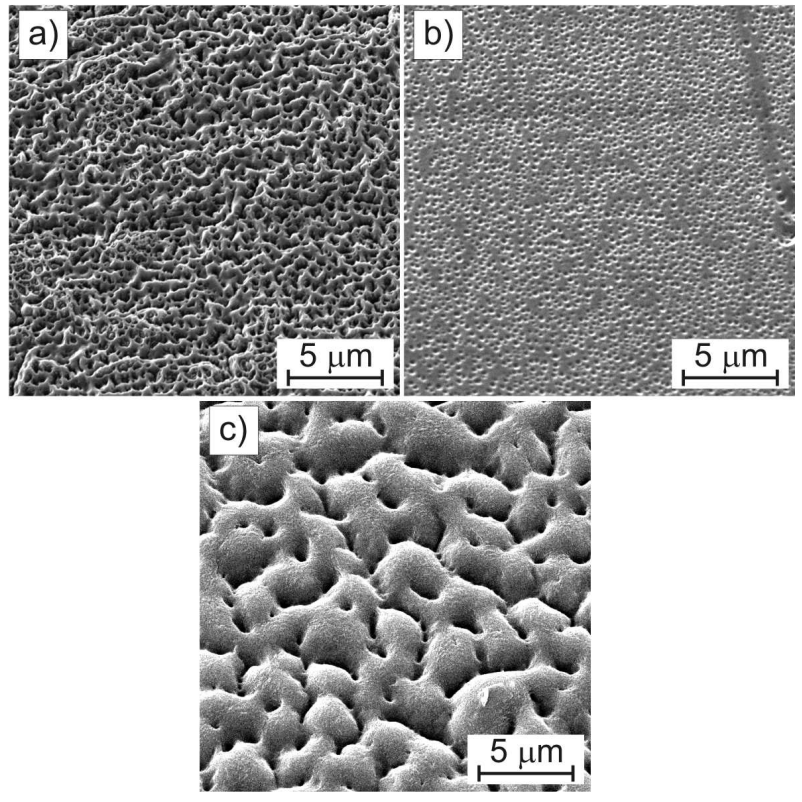

Fig. 8. SEM images of structures in polymers after 2 min EUV irradiation with the fluence close to the ablation threshold: a) PTFE, b) FEP, c) PMMA.

$5 \mu \mathrm{m}$ for $\mathrm{NaCl}$ and $1 \mu \mathrm{m}$ for $\mathrm{CaF}_{2}$. Similar structures were obtained also with smaller irradiation dose, delivered even within $10 \mathrm{~s}$ of exposure with $10 \mathrm{~Hz}$ repetition rate. The mechanism governing of fabrication of these structures is not clear. The attenuation length of EUV used for irradiation in $\mathrm{NaCl}$ is about $100 \mathrm{~nm}$ and about two times shorter in $\mathrm{CaF}_{2}$. Thus, the energy absorbed 

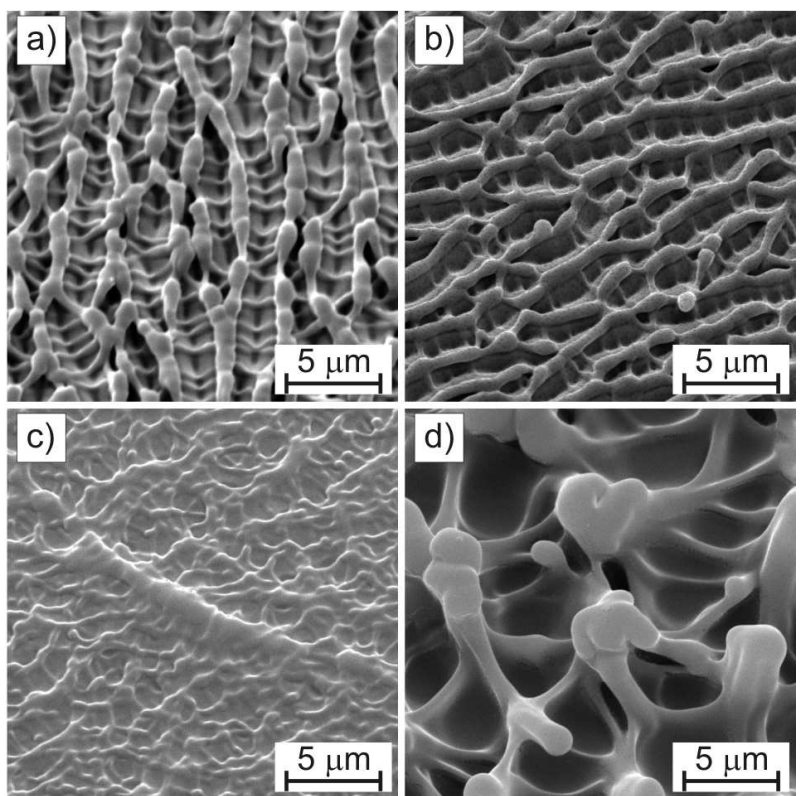

Fig. 9. SEM images of structures obtained in different polymers: a) PEN (25 EUV pulses), b) PET (25 EUV pulses), c) PVF (25 EUV pulses), d) PVDC (10 EUV pulses).
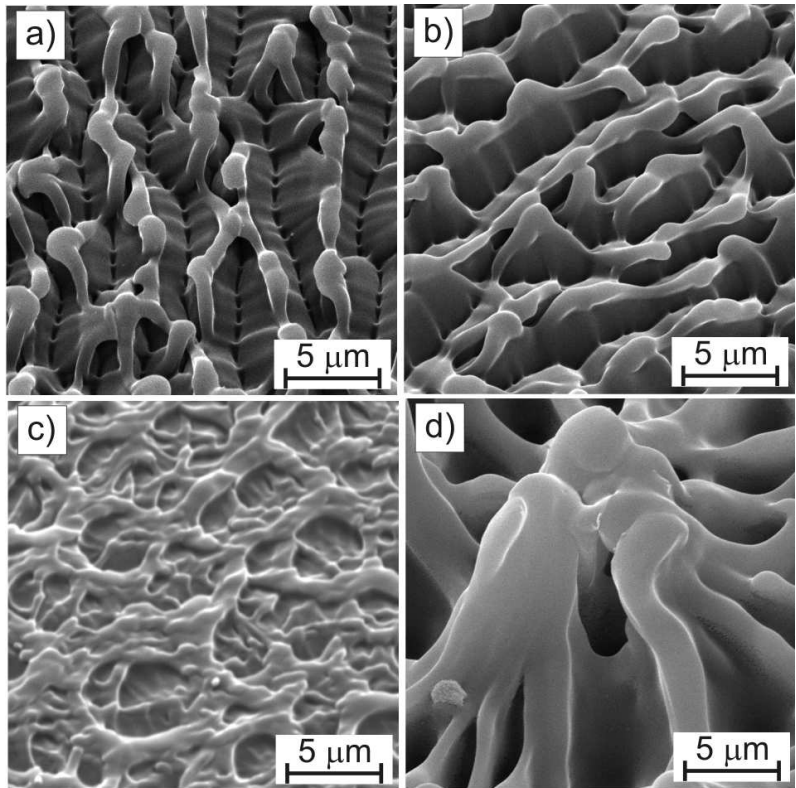

Fig. 10. SEM images of structures obtained in different polymers: a) PEN (50 EUV pulses), b) PET (50 EUV pulses), c) PVF (50 EUV pulses), d) PVDC (20 EUV pulses).

in a single shot is sufficient for melting of the layer. The microcrystalline structure is created probably as a result of fast melting followed by recrystallization. It may also arise due to stresses connected with the temperature gradient. Relatively large crystalline blocks obtained at the beginning can be divided during successive irradiation.
It can be also noticed that the crystalline structure in $\mathrm{CaF}_{2}$ after irradiation is not flat and there are no sharp edges in contrary to $\mathrm{NaCl}$ crystalline structure. It may be connected with a big difference in melting temperatures of both materials $-801^{\circ} \mathrm{C}$ for $\mathrm{NaCl}$ and $1418^{\circ} \mathrm{C}$ for $\mathrm{CaF}_{2}$. In the last case, solidification should be much faster and an amorphous layer may be formed on the surface of microcrystals. It seems possible that a proper choice of irradiation conditions could give nanocrystalline structure for some materials.


Fig. 11. SEM images of chemical compounds after 1 min EUV irradiation: a) $\mathrm{NaCl}$, b) $\mathrm{CaF}_{2}$.

a)

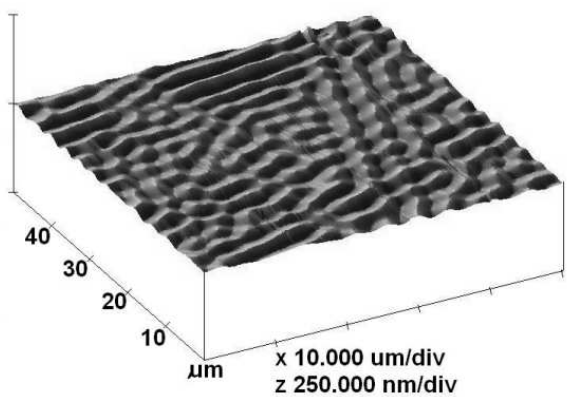

b)

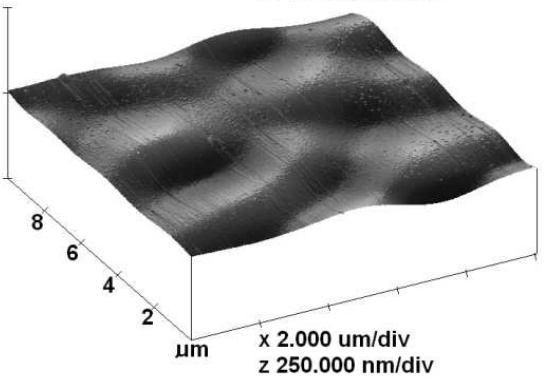

Fig. 12. AFM images of germanium after 2 min EUV irradiation - scan size: a) $50 \times 50 \mu \mathrm{m}$, b) $10 \times 10 \mu \mathrm{m}$.

Besides chemical compounds a germanium monocrystal was used in irradiation experiments. An attenuation length of EUV used for irradiation is about $30 \mathrm{~nm}$ and the melting point of germanium is $938.3^{\circ} \mathrm{C}$. A value of the melting point is not much higher comparing to $\mathrm{NaCl}$ so, it should be molten after irradiation. The main difference is that Ge is not a chemical compound and it can not be decomposed. It means that none of volatile components 
can be released and results of irradiation should be different. The Ge sample was mounted in the focal plane of the grazing incidence ellipsoidal collector and irradiated for $2 \mathrm{~min}$ with $10 \mathrm{~Hz}$ repetition rate. The surface structure after irradiation was investigated using a scanning electron microscope but SEM images with a good contrast could be obtained only under relatively small angle between the electron beam and the sample. That is why an atomic force microscope was employed for measurements of the Ge surface structure. Examples of AFM images of the resulting structure are shown in Fig. 12. In this case the structure was completely different as compared to $\mathrm{NaCl}$ and $\mathrm{CaF}_{2}$, as it was expected. A rippled structure with a height below $50 \mathrm{~nm}$ and the lateral size of approximately $5 \mu \mathrm{m}$ was generated. The surface was not cracked like it was for the chemical compounds. It may be some indication concerning an origin of cracks. They can be created as a result of the stress connected with releasing of volatile components inside a material. It would also explain the difference between $\mathrm{NaCl}$ and $\mathrm{CaF}_{2}$. A molecule of $\mathrm{CaF}_{2}$ contains two fluorine atoms that form a volatile medium after decomposition, while $\mathrm{NaCl}$ contains only one chlorine atom. That is why the amount of gas per volume released in $\mathrm{CaF}_{2}$ after irradiation would be greater than in $\mathrm{NaCl}$. It can result in smaller size of microcrystals formed on the $\mathrm{CaF}_{2}$ surface after irradiation.

\section{Conclusions}

In this paper we presented the results of experiments concerning micromachining, surface modification of organic polymers and interaction of EUV pulses with inorganic monocrystals. The experiments were performed using the laser-plasma EUV source based on the double stream gas-puff target. The source was equipped with two grazing incidence collectors of different type. Both collectors allowed to focus EUV in a wide spectral range with maximum centered at about $11 \mathrm{~nm}$ for Xe plasma and $10 \mathrm{~nm}$ for $\mathrm{Kr}$. It was shown that intensity of focused EUV was sufficient for ablation and surface modification of every organic polymers investigated in this work. It was also shown that micromachining of some polymers is possible and microstructures with the high aspect ratio were obtained. The surface modification was also obtained for three different inorganic monocrystals: $\mathrm{NaCl}$, $\mathrm{CaF}_{2}$ and $\mathrm{Ge}$ using the grazing incidence collector. Different kinds of surface structures were created in polymers depending on the EUV fluence. These were cones, ripples, pits and other more complicated structures in the micro- and nanoscale.

\section{Acknowledgments}

The research was partially performed in the frame of the EUREKA project E! 3892 ModPolEUV and under
COST Action MP0601, funded by the Ministry of Science and Higher Education (decision Nr 120/EUR/2007/02).

\section{References}

[1] Y. Zhang, T. Katoh, M. Washio, H. Yamada, S. Hamada, Appl. Phys. Lett. 67, 872 (1995).

[2] H. Fiedorowicz, A. Bartnik, L. Juha, K. Jungwirth, B. Králiková, J. Krása, P. Kubat, M. Pfeifer, L. Pína, P. Prchal, K. Rohlena, J. Skála, J. Ullschmied, M. Horvath, J. Wawer, J. All. Compd. 362, 67 (2004).

[3] H. Fiedorowicz, A. Bartnik, M. Bittner, L. Juha, J. Krasa, P. Kubat, J. Mikołajczyk, R. Rakowski, Micr. Eng. 73-74, 336 (2004).

[4] L. Juha, J. Krása, A. Cejnarová, D. Chvostová, V. Vorliček, J. Krzywiński, R. Sobierajski, A. Andrejczuk, M. Jurek, D. Klinger, H. Fiedorowicz, A. Bartnik, M. Pfeifer, P. Kubat, L. Pina, J. Kravárik, P. Kubeš, Y.L. Bakshaev, V.D. Korolev, A.S. Chernenko, M.I. Ivanov, M. Scholz, L. Ryć, J. Feldhaus, J. Ullschmied, F.P. Boody, Nucl. Instr. Meth. A 507, 577 (2003).

[5] A. Bartnik, H. Fiedorowicz, R. Jarocki, L. Juha, J. Kostecki, R. Rakowski, M. Szczurek, Micr. Eng. 78-79, 452 (2005).

[6] T. Makimura, Y. Kenmotsu, H. Miyamoto, H. Niino, K. Murakami, Surf. Sci. 593, 248 (2005).

[7] Y. Wan, Y. Wang, Z. Liu, X. Qu, B. Han, J. Bei, S. Wang, Biomaterials 26, 4453 (2005).

[8] E.K.F. Yim, R.M. Reano, S.W. Pang, A.F. Yee, C.S. Chen, K.W. Leong, Biomaterials 26, 5405 (2005).

[9] E. Rebollar, I. Frischauf, M. Olbrich, T. Peterbauer, S. Hering, J. Preiner, P. Hinterdorfer, C. Romanin, J. Heitz, Biomaterials 29, 1796 (2008).

[10] T. Gumpenberger, J. Heitz, D. Bauerle, H. Kahr, I. Graz, C. Romanin, V. Svorcik, F. Leisch, Biomaterials 24, 5139 (2003).

[11] V. Svorcik, K. Rockova, E. Ratajova, J. Heitz, N. Huber, D. Bauerle, L. Bacakova, B. Dvorankova, V. Hnatowicz, Nucl. Instrum. Meth. Phys. Research B 217, 307 (2004).

[12] A. Bartnik, H. Fiedorowicz, R. Jarocki, J. Kostecki, J. Mikołajczyk, R. Rakowski, M. Szczurek, in: Proc. SPIE, Vol. 5037, Eds. R.L. Engelstad, SPIE Press, Bellingham 2003.

[13] H. Fiedorowicz, A. Bartnik, R. Jarocki, R. Rakowski, M. Szczurek, Appl. Phys. B 70, 305 (2000).

[14] A. Bartnik, H. Fiedorowicz, R. Jarocki, L. Juha, J. Kostecki, R. Rakowski, M. Szczurek, Appl. Phys. B 82529 (2006).

[15] T. Lippert, Adv. Polym. Sci. 168, 51 (2004).

[16] B. Hopp, Zs. Bor, E. Homolya, E. Mihalik, Appl. Surf. Sci. 109/110, 232 (1997). 